\title{
ANALYSIS OF THE LEVEL OF CIRCULATING LEUKOCYTE PLATELET AGGREGATES IN WOMEN WITH BREAST CANCER
}

Maria Eduarda Borges Kerstenetzky1,2,3, Amanda Forte Mendes Tejo Salgado 1,2,3, Vitoria Ferreira David Melquiades ${ }^{1,2.3}$, Vinicius Rafael Agostinho Gomes, ${ }^{1,2,3}$, Denise Sobral Viana4, Jurema Telles de Oliveira Lima1, Marcelo Ramos Tejo Salgado ${ }^{4}$, Leuridan Cavalcante Torres ${ }^{1,2,4}$

IInstituto de Medicina Integral Professor Fernando Figueira - Recife (PE), Brazil. 2Prof CA Hart Translational Research Laboratory, Instituto de Medicina Integral Professor Fernando Figueira - Recife (PE), Brazil.

${ }^{3}$ Faculdade Pernambucana de Saúde - Recife (PE), Brazil.

${ }^{4}$ Pernambuco Cancer Hospital - Recife (PE), Brazil.

Recognition of support for the study: CNPq - National Council for Scientific and Technological Development, through the Institutional Program for Initiation Scholarships in Technological Development and Innovation (PIBITI).

Objective: To evaluate the levels of circulating platelet-leukocyte aggregates in women with breast cancer (BC). Methods: A cross-sectional study was carried out between 2018 and 2019 with 27 women, who aged between 18 and 60 years, diagnosed with BC and 15 healthy women (controls). For the evaluation of the circulating platelet aggregate, samples of peripheral blood were collected at the time of routine laboratory tests for diagnosis and before treatment. Platelet aggregate analysis was performed using monoclonal antibodies by flow cytometry. Mann-Whitney U and Kruskal-Wallis tests were used to analyze medians between two and three groups, respectively. Values of $\mathrm{p}<0.05$ were considered statistically significant. Analyses were performed on Graphpad v7.0. Results: In the analysis of the percentages of platelet-lymphocyte aggregates (AGP - lymphocytes) and platelet-neutrophils (AGP - neutrophils), no significant differences were observed between patients and controls. However, it was observed that the patients presented high percentage values of aggregate platelet-monocytes (AGP-monocytes) when compared with controls $(\mathrm{p}<0.0001)$. No significant differences were observed in the percentage levels of AGP-lymphocytes and AGP-neutrophils between the luminous subtypes A/B, HER2+, and triple-negative, and between these tumor subtypes and controls. The percentage values of AGP - monocytes were high in the luminous subtypes A/B, HER2+, and triple negative when compared with controls ( $\mathrm{p}=0.008,0.0001,0.0002$, respectively). However, no significant percentage differences in AGP-monocytes were observed between tumor subtypes. Conclusion: The present study showed the involvement of AGP-monocyte in triple-negative breast cancer and HER2+, these tumor subtypes being more aggressive and with a worse prognosis. Based on these data, the importance of new studies based on an investigation of the role of interactions between platelets and immune system cells in breast cancer became clear. The molecules involved in the linkages between platelets and leukocytes may be possible therapeutic targets.

Keywords: Breast Cancer; Platelets; Immune System. 